\title{
Téoros
}

Revue de recherche en tourisme

\section{Perceptions du Carnaval de Québec par les résidents de la région de Québec et par les touristes}

\section{Renée Dubé et Michel Zins}

Volume 12, numéro 1, mars 1993

Québec, capitale et région touristiques

URI : https://id.erudit.org/iderudit/1078200ar

DOI : https://doi.org/10.7202/1078200ar

Aller au sommaire du numéro

Éditeur(s)

Université du Québec à Montréal

ISSN

0712-8657 (imprimé)

1923-2705 (numérique)

Découvrir la revue

Citer cet article

Dubé, R. \& Zins, M. (1993). Perceptions du Carnaval de Québec par les résidents de la région de Québec et par les touristes. Téoros, 12(1), 32-35.

https://doi.org/10.7202/1078200ar d'utilisation que vous pouvez consulter en ligne. 


\section{Perceptions du Carnaval de Québec par les résidents de la région de Québec et par les touristes}

Renée Dubé et Michel Zins ${ }^{*}$

\section{Rappel historique}

Après la Russie et la NouvelleOrleans, apres Nice, Venise et Trinidad, après Rio et Montréal (qui a eu un carnaval de 1883 a 1889), Québec entrait dans la grande famille des fêtes populaires, le 29 janvier 1894, en inaugurant son premier camaval d'hiver.

Déjà a cette époque, le Carnaval associe les manifestations sportives aux rencontres sociales, un défilé nocturne, de la tradition, du folklore, de la fête et, bien sûr, le Palais de Glace. Cette activité semble, dès lors et déjà, profitable aux hôteliers, restaurateurs et commerçants de Québec.

Comme on le constate, il s"agit presque la d'une ébauche de la programmation de l'événement contemporain, avec ses manifestations sportives, ses rencontres sociales, ses activités culturelles et son défilé.

De 1894à 1954, le Camaval de Québec s'est tenu de façon irrégulière, intéressant et vivant mais toujours menacé. A chaque année, bien que répondant selon toute évidence aux attentes des gens, l'existence même de l'événement était remise en question. En 1954, le Carnaval prit la forme que nous lui connaissons actuellement, doté d'une organisation stable et structurée.

Entre la premiére (1954) et la plus récente (1993) édition du Carnaval deQuébec, l'événement a connu toutefois une douce transformation. En effet, un certain nombre d'événements et d'activités, quelques-uns à la fois, sont ajoutés ou retirés prudemment, tandis que certains autres sont déplacés d'un siteà un autre. On constate également $l^{\dagger}$ evolution du financement, une modernisation réfléchie du concept qui suit l'évolution des moeurs et des modes, bref une lente transition vers un produit social, culturel, récréatif et touristique complet, susceptible de plaire à la plus large clientèle, qu'elle soit d'origine locale ou touristique. Ilest possible d'en dire

Madame Renée Dubée. M Sc, est directrice. adjointe chez Zins Beauchesne et associes; monsieur Michel Zins est professeur à l"Université Laval et président de Zins Beauchesne et associbs. toutautant del'événement actuel, qui a connu une toute aussi grande fréquentation, et ce, malgre le froid...

L'évênement a aussi êté contesté et remis en doute cependant, par des critiques de la région de Québec elle-même. Les arguments étaient que cetévénement dérangeaitet visait uniquement les touristes; on concluait rapidement qu'il n'intéressait pas les gens de la région et que personne n'en était satisfait. Une étude menée pour valider ou infurmer ces critiques projette une toute autre image.

Une étude a donc été réalisée lors de l'édition de 1989 du Carnaval comprenant un sondage auprès de 300 adultes qui résidaient dans la région de Québec et qui ont participé au Camaval de Québec de 1989; un sondage auprès de 300 adultes qui résidaientau Québec mais à l'extérieur de la région de Québec et qui ont participé au Camaval de Québec de 1989; un sondage aupres de 200 adultes qui résidaient à l'extérieur de la province de Québec et qui ont participé au Camaval de Québec de 1989; un sondage auprès de 200 adultes quirésidaientdans larégiondeQuébec et qui n'ont pas participé au Camaval de Québec de 1989; un sondage auprès de 200 adultes qui résidaient dans la région de Montréal et qui n'ont pas participé au Carnaval de Québec de 1989.

Lasélection desrépondantsaétéeffectuéede façon aléatoire sur les lieux d'activitess. Les entrevues ont été récalisécs par téléphone sur une période de deux semaines suivant la sélection sur les lieux d'activités. (1)

\section{Clientèle de la région}

\section{La participation et la satisfaction}

Sur l'ensemble des adultes qui résident dans la région de Québec, $37 \%$ ont participé a au moins une activité organisée dans le cadre du Carnaval.

Sur les $65 \%$ des adultes de la région de Québec qui n'ont pas participé à l'édition 1989 du Carnaval de Québec, presque tous (soit $94 \%$ ) avaient déjă participé dans les annees antérieures.
Trois grands motifs de non-participation se dégagent: le manque d'intérêt $(29 \%)$ ), le manque de temps $(27 \%)$ et l'âge ou des problèmes de sante $(18 \%)$.

Quant aux motifs de participation, on peut conclure que $80 \%$ des participants trouvent leur propre intérêt dans cet événement tandis que pour $20 \%$, des motifs extemes tels la présence d'enfants ou la visite de parents justifient prioritairement la participation.

De plus, environ $80 \%$ des participants ont une perception plutôt favorable de l'évolution du Carnaval et y participent davantage ou tout autant au fil des ans. La plus large part de participation (59\%) s'observe chez les personnes âgées de 25 à 44 ans, mais elle se répartit assez bien entre les diverses catégories de scolarité, de revenu et d'emploi.

Le tableau 1 expose les taux de participation aux différentes activités du Carnaval de même que les taux de satisfaction liés à ces activités. La première colonne du tableau présente le pourcentage d'adultes interrogés qui ont participé à l'activité. La deuxiềne colonne présente le pourcentage de satisfaction pour les participants à l'activité qui regroupe les pourcentages de stres satisfait et satisfaits. Les cas de non-participation à l'activité ne sont pas considérés dans les pourcentages de satisfaction.

Les taux de satisfaction de la clientèle de la région sont très élevés dans l'ensemble. Il est a noter que $79 \%$ des participants se disent personnellement intéressés par le Camaval en tant que tel.

La perception du Carnaval de Québec

Le tableau 2 presente les pourcentages d'accord avec 23 énoncés proposés aux répondantset portantsurdivers aspects reliés au Camaval.

De façon générale, on peut conclure que l'évaluation du Camaval par les participants de la région de Québec est positive. La perception d'ensemble pourrait se resumer comme suit: le Carnaval de Québec est une grande fête populaire traditionnelle, ouverte 
à tous, qui contribue à animer la ville durant l'hiver et qui a l'avantage d'attirer des touristes dans la région et de générer des retombées intéressantes. Notons que cette perception est partagée par au moins $80 \%$ des participants. Par contre, sur certains aspects, l'avis des participants de Québec est nettement moins unanime.

Finalement, on soulignera que $25 \%$ des répondants interrogés trouveraient intéressant de participer comme bénćvoles a l'organisation du Carnaval, résultat assez évocateur d'une bonne perception.

\section{Perceptions face aux activités}

On a demandé aux répondants s'ils trouvaient qu'il y avait assez d'activités de différents types. Le tableau 3 expose les résultats.

Un participant sur deux évalue qu'il devrait y avoir plus d'activités de participation, ce qui est une proportion assez considérable. Plus d'un participant sur trois évalue pour sa part qu'il devrait y avoir plus d'activités familiales et d'activités culturelles.

Les intentions futures de participation au Carnaval sont très élevées et atteignent $88 \%$. Ceci tend a confirmer les taux de satisfaction relativement élevés qui se dégagent du sondage conduit auprès des participants de la région de Québec.

\section{Clientèle touristique}

\section{La participation et la satisfaction}

Parmi les Québecoisrésidanthorsde larégion de Québec, $68 \%$ des gens interrogés sur les sites étaient venus a Québec spécialement pour le Carnaval; parmi les non-Québécois interrogés durant l'événement, $76 \%$ étaient là spécialement pour le Carnaval. À lui seul, l'événement est un motif de déplacement important qui justifie des séjours dans la région de Québec en période hivernale.

Cependant, en 1989 , seulement $3,41 \%$ des adultes de la région de Montréal ont participé au Carnaval de Québec. Trois grands motifs de non-participation ressortent: le manque de temps $(37 \%)$, les obligations familiales ou de travail $(21 \%)$ et le manque d'interêt (19\%).

Quant aux touristes, les résultats tendent aussi à démontrer qu'à chaque année, le Carnaval attire une nouvelle clientèle touris-

\section{TABLEAU 1}

\section{Activités}

Visiter la Place du palais

Visiter le concours de sculpture sur neige

Visiter la Place de la famille

Acheter l'effigie du Carnaval

Acheter la bougic

Visiter la rue Sainte-Thérèse

Assister à la deuxième parade

Visiter la Foret Enchantée

Assister à la course en canot

Assister au toumoi de hockey Pee-Wee

Assister à la première parade

Visiter la Place des Arts

Participer à des activités de plein air du Carnaval

Assister au couronnement de la reine

Assister à une soirće du Camaval
82

59

80

\section{TABLEAU 2}

Clientèle de la région de Québec

$\begin{array}{ccc}\text { Énonee } & \% & \% \\ \text { accord } & \text { désaccord ne sais pas }\end{array}$

- Le Carnaval est un événement dont les gens de la région peuvent être fiers

- Le Carnaval de Québec est une

tradition qu'il faut respecter

96

3

Le Carnaval est une activité qui génère des retombées

95

5

intéressantes pour la région de Québec

- Le Carnaval est une grande fête où toute la famille peut $\mathrm{s}^{\prime}$ amuser

- Le Carnaval est une activité sans laquelle l'hiver serait morne à Québec

- Durant les activités du Carnaval, lodre et la sécurité publique sont correctement assurés

- Le Carnaval offre un ensemble d'activités intéressantes pour tout le monde

- Le Camaval a fait de grands efforts récemment pour amener les participants à plus de civisme

- Le Camaval, c'est bruyant

- Le Carnaval, c'est toujours pareil

- Il manque de musique d'ambiance sur les sites

- Le Carnaval n'est pas fait pour les personnes âgées

- Le Carnaval manque d'activités de jour

- Le Carnaval est une activité principalement conçue pour les jeunes

- Je trouverais intéressant de participer comme bénévole à l'organisation du Carnaval

- Le Carnaval n'est qu'une grande beuverie

- Durant le Carnaval, la ville est malpropre

- La programmation des activités n'est pas assez variée

- Le Camaval manque d'activités le soir

- Le Carnaval est plus intéressant pour les gens de la ville que pour les touristes

- Le Carnaval dérange la population de la grande région de Québec

- Le Camaval me dérange personnellement dans mes activités

- Le Carnaval attire trop de touristes

87

6

12

83

16

82

48

43

43

34

30

28

25

25

23

19

14

12

9

2

3 
tique: $65 \%$ des gens interrogés venant du reste de la province en étaient à leur première participation en 1989 et cette proportion atteint $84 \%$ chez la clientèle touristique hors Québec.

Le tableau 4 expose les taux de participation aux differentes activités du Camaval, de même que les taux de satisfaction liés à ces activités pour les participants du reste de la province et pour les touristes de l'extérieur de la province. Le pourcentage de satisfaction regroupe les catégories: atrès satisfait et «satisfaito. Les cas de non-participation à l'activité ne sont pas considérés dans les pourcentages de satisfaction.

Au plan de la participation, deux activités dominent nettement: visiter la Place du Palais $(97 \%)$ et visiter le concours de sculpture sur neige en face du manè ge militaire $(83 \%$, $80 \%$ ). Dans un deuxième bloc, on retrouve deux activités qui atteignent des taux de participation excédant $50 \%$ : visiter la rue Sainte-Thérèse et acheter l'effigie du Carnaval.

A propos des taux de satisfaction des participants, ils sont élevés dans l'ensemble ( $90 \%$ et plus). Aucun problème ne ressort sur ce plan. On remarque que la participation décroît avec l'âge après 35 ans. La plus large part de participation s'observe entre 25 et 34 ans $(32 \%, 33 \%)$.

\section{La perception du Carnaval de Québec par les touristes}

Le tableau 5 présente les pourcentages d'accorden rapportavec 21 énoncés proposés aux répondants portant sur divers aspects relies au Camaval.

On peut encore une fois conclure que l'évaluation du Camaval par les participants du reste de la province et par les touristes de l'extérieur de la province est positive.

De plus, les intentions futures de participation au Camaval sont très élevées (74\%), considérant que cette clientèle touristique doitse déplacer pour participerà l'événement. Ceci tendà confirmer les taux de satisfaction relativement élevés qui ressortent du sondage conduit auprès des touristes québćcois de l'extérieur de la région.

Les participants du reste de la province sont venus à Québec avec un groupe d'amis (33 $\%)$, en couple $(30 \%)$ ou avec leur conjoint et leurs enfants $(30 \%)$.

\begin{tabular}{|c|c|c|c|c|}
\hline \multicolumn{5}{|c|}{ TABLEAU 3} \\
\hline & \multicolumn{4}{|c|}{ Clientèle de la région de Québec } \\
\hline & Types d'activités. & $\begin{array}{l}\text { Pas assez } \\
\text { d'activités }\end{array}$ & $\begin{array}{c}\text { Assez } \\
\text { d'activités }\end{array}$ & $\begin{array}{l}\text { Trop } \\
\text { d'activites }\end{array}$ \\
\hline & Activités de participation, comme du ski & & & \\
\hline E & $\begin{array}{l}\text { de fond, un marathon pour le grand public, etc. } \\
\text { Activités familiales }\end{array}$ & 49 & $\begin{array}{l}51 \\
50\end{array}$ & 1 \\
\hline - & Activités culturelles & 39 & 61 & - \\
\hline . & Activités sportives & 27 & 71 & 3 \\
\hline - & Activités de participation de masse (ex.: parade & 16 & 82 & 2 \\
\hline . & Activités sociales comme des soirées et des bals & 15 & 80 & 6 \\
\hline
\end{tabular}

TABLEAU 4

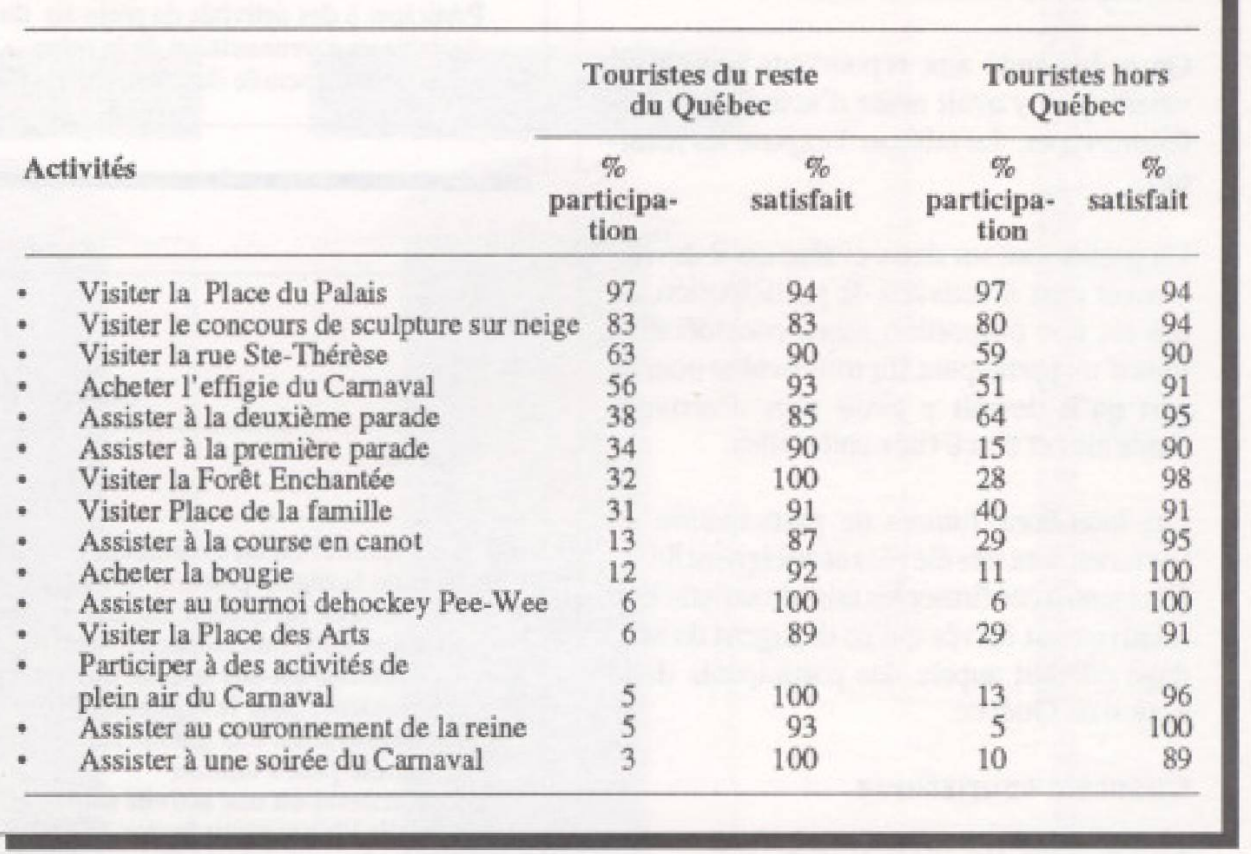

L'automobile est de loin lemode de transport le plus fréquemment utilisé $(91 \%)$ par les touristes du reste de la province. L'avion et le train ont des taux d'utilisation très faibles voire nuls.

Pour deux touristes sur trois qui ont passé au moins une nuit à Québec pour le Camaval $(77 \%)$, le mode d'hébergement utilisé est la résidence de parents ou d'amis, L'hếbergement commercial est utilisé dans un cas sur trois.

Les participants de l'extérieur de la province sont surtout venus a Québec avec un groupe d'amis (39\%) ou en couple (31\%). A noter que $15 \%$ sont venus avecun groupe organise.

L'automobile est le mode de transport le plus fréquemment utilisé: dans un cas sur deux. L'autobus suit au second rang avec un participant sur quatre qui l'a utilisé. L'avion et le train viennent aux derniers rangs avec des taux d'utilisation respectifs de $17 \%$ et $11 \%$.

Pour $80 \%$ des touristes qui ont passé au moins une nuit à Québec pour le Carnaval, le mode d'hébergement utilisé est l'hébergement commercial.

Les non-participants

de la région de Québec

On relève trois principaux motifs de nonparticipation au Carnaval de 1989: le manque d'intérêt $(29 \%)$ et le manque de temps $(27 \%)$, de même que les obligations familiales pour près de deux répondants sur dix.

La très grande majorité des répondants sot 94 $\%$ ont déjà participé au Carnaval dans le passé tandis que seulement $7 \%$ n'y ont jamais participé. 


\section{TABLEAU 5}

\begin{tabular}{|c|c|c|c|c|c|c|}
\hline \multirow[b]{2}{*}{ Énoncé } & \multicolumn{3}{|c|}{ Touristes du reste du Québec } & \multicolumn{2}{|c|}{ Touristes hors Québec } & \multirow[b]{2}{*}{$\begin{array}{c}\% \\
\text { ne sais pas }\end{array}$} \\
\hline & $\begin{array}{c}\% \\
\text { accord }\end{array}$ & $\begin{array}{c}\% \\
\text { désaccord }\end{array}$ & $\begin{array}{c}\% \\
\text { ne sais pas }\end{array}$ & $\begin{array}{c}\% \\
\text { accord }\end{array}$ & $\begin{array}{c}\% \\
\text { désaccord }\end{array}$ & \\
\hline \\
\hline $\begin{array}{l}\text { qu'il faut respecter } \\
\text { Le Carnaval est un événement dont les }\end{array}$ & 99 & 1 & - & 99 & 1 & - \\
\hline gens de la région peuvent être fiers & 98 & 1 & 1 & 98 & 1 & 1 \\
\hline Le Carnaval est une grande fete où & & & & & & \\
\hline toute la famille peut s'amuser & 97 & 3 & - & 99 & 1 & - \\
\hline interessantes pour tout le monde & 93 & 5 & 2 & 98 & 2 & 1 \\
\hline Durant les activités du Camaval, l'ordre et la & & & & & & \\
\hline $\begin{array}{l}\text { sécurité publique sont correctement assurés } \\
\text { Le Carnaval est une activité sans }\end{array}$ & 84 & 11 & 5 & 89 & 8 & 3 \\
\hline laquelle l'hiver serait morne à Québec & 80 & 16 & 4 & 65 & 25 & 10 \\
\hline Le Carnaval a fait de grands efforts récemment & & & & & & \\
\hline pour amener les participants à plus de civisme & 54 & 3 & 42 & 45 & 4 & 51 \\
\hline Le Carnaval, c'est bruyant & 42 & 57 & 1 & 33 & 67 & - \\
\hline Il manque de musique d'ambiance sur les sites & 40 & 55 & 6 & 23 & 74 & 4-. \\
\hline Le Carnaval c'est toujours pareil & 26 & 29 & 45 & 16 & 21 & 64 \\
\hline Le Carnaval manque d'activités le jour & 25 & 56 & 19 & 30 & 59 & 11 \\
\hline Le Carnaval n'est qu'une grande beuverie & 24 & 73 & 3 & 19 & 80 & 2 \\
\hline Le Carnaval n'est pas fait pour les personnes ågées & 18 & 78 & 5 & 39 & 57 & 5 \\
\hline La programmation des activités n'est pas assez variée & 16 & 67 & 18 & 16 & 75 & 10 \\
\hline $\begin{array}{l}\text { Le Camaval est une activité } \\
\text { principalement concue pour les jeunes }\end{array}$ & & 83 & & & & \\
\hline Durant le Carnaval. la ville est malpropre & 13 & 86 & 1 & 10 & $\begin{array}{l}83 \\
90\end{array}$ & - \\
\hline Le Carnaval manque d'activités le soir & 9 & 75 & 15 & 9 & 82 & 10 \\
\hline $\begin{array}{l}\text { Le Carnaval dérange la population de la } \\
\text { grande région de Ouébec }\end{array}$ & 9 & 60 & 31 & 11 & 54 & 35 \\
\hline Le Carnaval est plus intéressant pour & 8 & 00 & 31 & 11 & 34 & 35 \\
\hline les gens de la ville que pour les touristes & 5 & 88 & 7 & 11 & 81 & 9 \\
\hline Le Carnaval attire trop de touristes & 3 & 95 & 1 & 5 & 89 & 6 \\
\hline $\begin{array}{l}\text { Le Carnaval me dérange personnellement } \\
\text { dans mes activités }\end{array}$ & 2 & 98 & - & 1 & 97 & 2 \\
\hline
\end{tabular}

Les non-participants de la région de Québec sont unanimes pour reconnaitre certaines forces du Carnaval de Québec. A ceteffet, on peut résumer la perception positive comme suit: le Carnaval de Québec est une grande fêtepopulaire traditionnelle, où tout le monde peut s'amuser, qui contribue à animer la ville durant l'hiver et qui al'avantage d'attirer des touristes dans la région et de générer des retombées intéressantes. On note aussi qu'à peu près toutes les personnes interrogées mentionnent que le Carnaval n' attire pas trop de touristes. $A$ noter que cette perception positive est celle d'au moins $80 \%$ des nonparticipants de Québec.

Finalement, il faut noter que $15 \%$ des répondants interrogés trouveraient intéressant de participer comme bénévoles a l'organisation du Camaval.

\section{Les non-participants de la région de Montréal}

On a demandé aux non-participants de Montréal s'ils avaient dêjả participé au Camaval de Québec par le passé. Plus de la moitié des répondants, soit $52 \%$, ont deja participé tandis que $48 \%$ n'y ont jamais participé.

On releve trois principaux motifs de nonparticipation au Carnaval de 1989: le manque de temps pour près de deux répondants sur cinq, les obligations familiales ou de travail et le manque d'intérêt pour un répondant sur cinq.

De façon générale, on peut conclure que l'evaluation du Carnaval par les non-participants de la région de Montréal est positive.

\section{Conclusion}

Il apparaît donc clairement, à la lecture des résultats de cette étude, que certains constats positifs font l' unanimité autant chez les participants que les non-participants et peu importe l'origine: le Carnaval est unévénement dont les gens de la région peuvent être fiers; le Camaval de Québec est une tradition qu'il faut respecter; le Carnavalest une activité qui génère des retombées intéressantes pour la régionde Québec; le Camaval est une grande fête où toute la famille peut s'amuser; le Camaval est une activité sans laquelle l' hiver serait momeàQuébec; durant les activités du Camaval, l'ordre et la sćcurité publique sont correctement assurés; le Carnaval a fait de grands efforts récemment pour amener les participants à plus de civisme; le Carnaval est intéressant pour les gens de la ville et pour les touristes.

Contrairement à certaines idées qui circulent, le Carnaval est donc perçu de façon unanime comme une grande fête traditionnelle qui contribue à animer la ville et qui a l'avantage d"attirer des touristes dans la région et de générer des retombées intéressantes.

Les parades, les sculptures et monuments, le Palais de glace et l'ambiance générale sont des forces indéniables de l'événement

\section{RÉFÉRENCES}

(1) Zins Beauchesne ot associés (1989), Etude do planification stratégique du Carnaval de Qubbec : 1990-1995. 